\title{
Expression of endothelin-1 in laryngocarcinoma tissues and its clinical significance
}

\author{
SHAOZHONG WANG ${ }^{1}$, JIAN WU ${ }^{2}$, YISA SONG ${ }^{1}$ and HUILING ZHONG ${ }^{1}$ \\ ${ }^{1}$ Otorhinolaryngology Department of Qinghai People's Hospital, Xining, Xining, Qinghai 810007; \\ ${ }^{2}$ Department of Otolaryngology and Head and Neck Surgery, Changzheng Hospital, \\ The Second Military Medical University, Shanghai 200003, P.R. China
}

Received December 29, 2015; Accepted April 4, 2016

DOI: $10.3892 / \mathrm{ol} .2016 .4406$

\begin{abstract}
In recent years, the incidence of laryngeal carcinoma has been on the increase. The aim of the present study was to examine the expression level of endothelin-1 (ET-1) in laryngeal carcinoma tissues and to establish its clinical significance. A total of 82 cases of laryngeal carcinoma tissues were examined, of which 27 cases were stage I, 34 were stage II, and 21 were stage III. The normal mucosal tissues beyond the surgical incision margin in the 82 laryngeal carcinoma patients were used as the normal control. An additional 80 tissue specimens collected from laryngeal carcinoma outpatients were used as benign lesions. ET-1 expression levels in different tissues were determined using streptavidin-peroxidase (SP) immunohistochemistry. The results showed that the ET-1 expression level was highest in laryngeal carcinoma tissues and was significantly higher than that in the other two groups $(\mathrm{P}<0.05)$. The ET- 1 expression level was higher in stage III compared to that in stage I and II $(\mathrm{P}<0.05)$ and higher than that of the normal control $(\mathrm{P}<0.05)$. The ET-1 expression level was higher in stage II compared to that in stage I $(\mathrm{P}<0.05)$. In conclusion, ET-1 was strongly expressed in laryngeal carcinoma tissues and may play an important role in the pathogenesis and development of laryngeal carcinoma tissues. ET-1 expression in laryngeal carcinoma tissues was associated with the clinical staging of laryngeal carcinoma.
\end{abstract}

\section{Introduction}

Persistent global atmospheric pollution and lifestyle habits have led to an increase in the incidence of laryngeal

Correspondence to: Jian $\mathrm{Wu}$, Department of Otolaryngology and Head and Neck Surgery, Changzheng Hospital, The Second Military Medical University, 417 Fengyang Road, Shanghai 200003, P.R. China

E-mail:k1606y@163.com

Keywords: laryngocarcinoma, endothelin-1,immunohistochemistry carcinoma or laryngeal cancer (1). Consequently, laryngeal carcinoma constitutes a health burden, affecting quality of life. Malignant tumors are caused by multiple gene mutations and are regulated by multiple factors (1). Multi-level and multi-target therapies are usually administered for malignant tumors. Specific treatment depends on the site, type, and stage of the tumor. Treatment may involve surgery, radiotherapy, or chemotherapy, alone or in combination. This is a specialised area which requires the coordinated expertise of ear, nose and throat (ENT) surgeons (otolaryngologists) and oncologists. A severely affected patient may require a laryngectomy, the complete or partial removal of the vocal cords (1). However, more effective treatments should be identified. Therefore, a search for a biological system that can regulate laryngeal carcinoma growth and identification of new treatment targets has become a priority for the effective treatment of laryngeal carcinoma.

Endothelin (ET) is a bioactive peptide produced by endothelial cells that can constrict vessels vigorously (2). It can also enhance the constriction of myocardium and smooth muscles, and promote neuroendocrine. ET is a powerful pro-differentiation agent and a cell growth factor that can promote cell mitosis, participate in tumor growth and induce mitosis in tumor growth (3). Three types of ET have been identified, ET-1, ET-2 and ET3, of which ET-1 is the most potent biomolecule. It has been shown that ET-1 plays a major role in the regulation of the pathogenesis of malignant tumors (4). However, the relationship between ET and laryngeal carcinoma remains to be elucidated.

To gain a better understanding of the association between ET and laryngeal cancer, we studied the expression levels of ET in laryngeal carcinoma tissues using immunohistochemistry and examined the possible role of ET in the pathogenesis of laryngeal carcinoma.

\section{Patients and methods}

Materials. From January, 2005 to April, 2010, tumor samples were collected from 82 laryngeal carcinoma patients. There were 49 men and 33 females, with an age range of 27-76 years (mean, 46.51 \pm 6.51 years). All the patients participating in the present study underwent pre-operative pathological confirmation to ensure they had not previously undergone 
chemotherapy. Clinical pathological staging was performed according to the tumor node metastasis (TNM) classification and staging protocol. Of the 82 cases included in the study 27 cases were stage I, 34 were stage II, and 21 were stage III. The normal mucosal tissues beyond the surgical incision margin in the 82 laryngeal carcinoma patients were used as the normal control. No tumor invasion was detected in those samples. An additional 80 tissue specimens, including 51 cases of chorditis nodulosa and 29 vocal cord polyps, were collected from laryngeal carcinoma outpatients and were used as benign lesions. The benign lesions were confirmed by pathological examination.

The study protocol was approved by the Ethics Committee of Changzheng Hospital (Shanghai, China). Patients provided written informed consent.

Methods. Sample collection and preparation were performed by one investigator. The samples were rapidly fixed with $100 \mathrm{ml} / 1$ neutral formaldehyde solution, embedded in paraffin, and stored at $4^{\circ} \mathrm{C}$. The specimens were cut into $4 \mu \mathrm{m}$ sections and the sections were detoxified and stained with immunohistochemical streptavidin-peroxidase (SP) staining, incubated in citrate buffer (ZsBio, Beijing, China), and heated in a microwave (Joyn, Shanghai, China). The sections were stained with DAB (Boster, Wuhan, China), followed by re-staining with hematoxylin. Human arterial endothelial cell sections with a positive expression of ET-1 were used as the positive control, whereas tumor cell sections that were treated with PBS instead of primary antibody were used as the negative control. These sections were observed under an optical microscope (BX51, Olympus, Tokoyo, Japan) subsequent to immunohistochemical staining. Positively stained cells were brown, the nucleus stained with hematoxylin was blue, and the cytosol of negatively stained cell was colorless. Five fields were selected randomly under a 400x field. A pathological image analysis system was used to draw the cell outlines and calculate the area ratio of positive area and the whole labeled area.

Statistical analysis. Measurement data were presented as mean \pm standard deviation. The data were initially examined using the F-test, followed by the q-test. The paired data were assessed using the paired Student's t-test. Statistical analysis was performed with SPSS 13.0 software. $\mathrm{P}<0.05$ was considered statistically significantly.

\section{Results}

Comparison of the positive expression rate of ET-1 in the three groups. The ET-1 expression level in the laryngeal carcinoma group $(49.2 \pm 11.5)$ was significantly higher than those in the remaining two groups $(17.5 \pm 4.1$ and $6.2 \pm 2.4$, respectively). The differences were statistically significant $(\mathrm{P}<0.05)$. The ET-1 expression level in the benign lesion group was higher than that in the normal control group and the difference was statistically significant $(\mathrm{P}<0.05$; Table I).

Comparison of the positive expression rate in different TNM staging of laryngeal carcinoma. The highest ET-1 expression level was detected in stage III laryngeal carcinoma, whereas
Table I. Comparison of the positive expression rate of ET-1 in three groups.

\begin{tabular}{lccc}
\hline Group & $\mathrm{n}$ & Positive & $\begin{array}{c}\text { Rate of positive } \\
\text { expression }(\%)\end{array}$ \\
\hline Laryngeal carcinoma & 82 & 77 & $49.2 \pm 11.5^{\mathrm{a}, \mathrm{b}}$ \\
Benign lesion & 80 & 27 & $17.5 \pm 4.1^{\mathrm{a}}$ \\
Normal control & 82 & 9 & $6.2 \pm 2.4$ \\
\hline
\end{tabular}

${ }^{\mathrm{a}} \mathrm{P}<0.05$ vs. normal control group; ${ }^{\mathrm{b}} \mathrm{P}<0.05$ vs. benign lesion group. ET-1, endothelin-1.

Table II. Comparison of the positive expression rate in different TNM staging of laryngocarcinoma.

\begin{tabular}{lccc}
\hline Laryngeal carcinoma & $\mathrm{n}$ & Positive & $\begin{array}{c}\text { Rate of positive } \\
\text { expression (\%) }\end{array}$ \\
\hline Stage & & & \\
I & 27 & 23 & $28.1 \pm 8.2^{\mathrm{a}, \mathrm{b}}$ \\
II & 34 & 33 & $47.9 \pm 10.7^{\mathrm{a}}$ \\
III & 21 & 21 & $78.2 \pm 18.3$ \\
\hline
\end{tabular}

${ }^{\text {a }} \mathrm{P}<0.05$ vs. III stage; ${ }^{\text {}} \mathrm{P}<0.05$ vs. II stage. TNM, tumor node metastasis.

the lowest expression level was identified in stage I laryngeal carcinoma. The expression level in stage III (78.2 \pm 18.3$)$ was significantly higher than that identified in stage I and II (28.1 \pm 8.2 and 47.9 \pm 10.7 , respectively). The differences were statistically significant $(\mathrm{P}<0.05)$. The ET-1 expression level in stage II was considerably higher than that in stage I, and the difference was statistically significant $(\mathrm{P}<0.05$; Table II).

\section{Discussion}

Laryngeal carcinoma exhibits a high incidence in head and neck tumors and constitutes a health burden, affecting quality of life (5). A link exists between the ET system and the occurrence of malignant head and neck tumors. Although the number of studies on this subject is limited, results from existing studies have demonstrated that ET-1 promoted growth in laryngeal carcinoma cells (6). Further investigation is required to gain a better understanding of the association of ET with laryngeal carcinoma. To investigate the possible connection between the ET system and the occurrence of malignant head and neck tumors, we verified the ET expression levels in laryngeal carcinoma cells and studied the effect of ET-1 on the cell growth, apoptosis, metastasis and invasiveness of laryngeal carcinoma. We also investigated the possibility of using ET receptor antagonism to block the biological effects of ET-1 on the development of laryngeal carcinoma.

ET is a powerful pro-differentiation agent and multifunctional cell growth factor. ET promotes cell mitosis, is involved in the growth of multiple tumors and induces angiogenesis $(7,8)$. It also has cytokine-like effects, influences cellular 
DNA synthesis and positively affects the expression of protooncogenes and cell proliferation. Previous findings (9) have shown that ET synergized with other growth factors to activate proto-oncogenes and promoted cell mitosis and proliferation. Thus, it plays an important role in the pathogenesis and tumor development (10). Radioimmunoassay confirmed the presence of ET-1 in lung, renal, breast, and pancreatic cancers (11), while other studies revealed a relationship between ET-1 and tumor development (12). In the present study, we found that the expression levels of ET-1 were significantly higher in laryngeal benign lesion tissue compared to those in laryngeal normal tissue $(\mathrm{P}<0.05)$. This indicated that ET-1 may be involved in the pathogenesis of laryngeal benign lesion. The expression level of ET-1 was highest in laryngeal carcinoma tissue and significantly higher than that in laryngeal benign lesion tissue and normal control tissue $(\mathrm{P}<0.05)$. These results confirmed that ET-1 was strongly expressed in tumor tissues, and indicated that ET-1 likely played an important role in laryngeal carcinoma pathogenesis.

Comparative studies on the staging and quantification of lung squamous cell carcinoma and adenocarcinoma, demonstrated that higher differentiation in tumor tissues inversely correlated with decreased levels of ET-1. Those findings suggested that ET-1 expression level was adversely proportional to the differentiation of lung cancer tissue, and further predicted that the expression level of ET-1 was proportional to the malignancy of lung cancer tissue (13). The present findings have demonstrated that the ET expression level was highest in stage III and lowest in stage I, while the ET expression level in III stage was significantly higher than that in stage I and II $(\mathrm{P}<0.05)$. In addition, the ET expression level in stage II was significantly higher than that in stage I $(\mathrm{P}<0.05)$. ET expression level was noteworthy in tumors in higher stages, which suggested that that ET expression level was closely associated with the clinical staging of laryngeal carcinoma. Furthermore, the ET expression level indicated the malignancy and prognosis of laryngeal tumor, which was consistent with previous literatures (14).

In conclusion, the results of the present study demonstrate ET-1 was closely associated with laryngeal tumor. ET-1 was highly expressed in laryngeal carcinoma and possibly played an important role in the pathogenesis and development of laryngeal carcinoma. In addition, the expression of ET-1 was associated with the clinical staging of laryngeal carcinoma. The results provide theoretical fundamental to further investigate the connection between ET-1 and the pathogenesis and development of laryngeal carcinoma.

\section{References}

1. Uva P, Aurisicchio L, Watters J, Loboda A, Kulkarni A, Castle J, Palombo F, Viti V, Mesiti G, Zappulli V, et al: Comparative expression pathway analysis of human and canine mammary tumors. BMC Genomics 10: 135, 2009.

2. Davenport AP, Hyndman KA, Dhaun N, Southan C, Kohan DE, Pollock JS, Pollock DM, Webb DJ and Maguire JJ: Endothelin. Pharmacol Rev 68: 357-418, 2016.

3. Flammer J and Konieczka K: Retinal venous pressure: The role of endothelin. EPMA J 6: 21, 2015.

4. Li C, Sun Y and Liu B: The relationship between endothelin and tumor. Foreign Med Oncol Branch 30: 360-362, 2003.

5. Marchiano E, Chin OY, Fang CH, Park RC, Baredes S and Eloy JA: Laryngeal adenoid cystic carcinoma: A systematic review. Otolaryngol Head Neck Surg 2154: 433-439, 2016.

6. Rosanò L1, Spinella F, Di Castro V, Nicotra MR, Dedhar S, de Herreros AG, Natali PG and Bagnato A: Endothelin-1 promotes epithelial-to-mesenchymal transition in human ovarian cancer cells. Cancer Res 65: 11649-11657, 2005.

7. Chen MS, Yu WB, Fan YH, Zhanc XN and Ma LC: Detection of serum endothelin contents in patients with malignant tumors and its clinical significance. Xi Bao Yu Fen Zi Mian Yi Xue Za Zhi 17: 40-41, 2001 (In Chinese).

8. Tan H, Li L and Zhang S: The relationship between endothelin-1 and tumor angiogenesis. J Glob Tumor 3: 100-103, 2004.

9. Lien CC, Jiang JL, Jian DY, Kwok CF, Ho LT and Juan CC: Chronic endothelin-1 infusion causes adipocyte hyperplasia in rats. Obesity (Silver Spring) 24: 643-653, 2016.

10. Qi HW, Ye JF, Song LQ, Chen WQ, Liang J and Mu DG: Exploration of serum endothelin-1 as a marker of early radiation lung injury. Xi Bao Yu Fen Zi Mian Yi Xue Za Zhi 21: 356-358 2005.

11. Xu D, Li Z, Wu B, et al: The expression of endothelin system in laryngocarcinoma cell HEP2. Proc Med Postgrad 23: 21-24, 2010.

12. Teng XJ, Shen ZX, Xiang JJ, Shen L, Yuan L, Guo J and Wang XL: Peripheral plasma Big endothelin 1 levels in patients with gastric carcinoma undergoing radical gastrectomy and its relationship with tumor recurrence. Zhonghua Wei Chang Wai Ke Za Zhi 9: 34-37, 2006 (In Chinese).

13. Hagemann T, Binder C, Binder L, Pukrop T, Trümper L and Grimshaw MJ: Expression of endothelins and their receptors promotes an invasive phenotype of breast tumor cells but is insufficient to induce invasion in benign cells. DNA Cell Biol 24: 766-776, 2005.

14. Zhang J, Zhong W, Luo W and Zhang Z: Expression of Endothelin-1 in laryngeal carcinoma. J Clin Otolaryngol 20: 1124-1125, 2006 (In Chinese). 